\title{
Background problems in optical transient searches
}

\section{R. Hudec}

Astronomical Institute, CZ-251 65 Ondřejov, Czech Republic

Received January 21; accepted May 10, 1999

\begin{abstract}
The optical transient (OT) searches are heavily affected, especially at low magnitudes, by background. I will discuss the most important sources of this background, and suggest a suitable strategy for searches for optical afterglows of GRBs in optical.
\end{abstract}

Key words: gamma-ray: bursts

\section{Introduction}

Despite exciting new results (7 GRB-related optical afterglows (OAs) discovered), there still remain questions: more than one fading optical object has been revealed in or close to GRB boxes in several cases and, in one case, more than one X-ray fading source has been found in GRB box. For GRB 970616, two X-ray fading counterparts and two optically fading objects, one inside gamma ray box but both outside X-ray boxes have been detected (Greiner et al. 1997). For GRB 970402, an optically variable/fading source was found outside the X-ray error box. The gamma-ray error box of GRB 970508 exhibits several optical variable sources, only one in the X-ray box (Djorgovski et al. 1997). There are two X-ray sources in the GRB 980425 error box. The fading X-ray source is not coinciding with optical and radio source (SN 1998bw). The obvious conclusion may be drawn, namely that not all optical and probably also not all X-ray variable/fading objects found at or close to the GRB positions are related to GRBs in question. There is a background of variable optical (and also X-ray ) sources not related to GRBs. This background is especially important for large error boxes.

Let us try to summarize what have we learned from the Optical Afterglows (OA) analyses: (1) 7 OAs were detected from 19 GRBs studied i.e. $\sim 40 \%$ of GRBs exhibit optical emission (2) they peak at $18-23 \mathrm{mag}(3)$ they exhibit power law declines with mean power of -1.2 (4) they seem to be related to faint host galaxies (but not

Send offprint requests to: R. Hudec all) (5) not all GRBs have an OA (6) the optical emission less than 3 hrs after GRBs remains poorly investigated (7) the direct optical emission is below $V=7-10$ mag (Hudec et al. 1999) (8) at least some OAs are very likely associated with GRBs e.g. due to excellent positional and temporal coincidence and common power law fading.

One can conclude that GRBs may be monitored and studied by observing their OAs. This may open a new observing window for monitoring GRBs. Further, the rate of OAs may be higher than the rate of GRBs due to different beaming, the factor is unknown but may be up to 100 , hence such studies require good knowledge of OT related background (false events not related to GRBs but with similar transient behavior)

\section{The background sources}

\subsection{Supernovae}

The supernovae, especially those of Ia type, may represent an important source of confusion due their occurrence rates, rise and decay timescales, and magnitudes. The expected rate of occurrence for faint events (down to $R 23 \mathrm{mag}$ ) is roughly $2 \mathrm{deg}^{-2}$ or $0.0015 \operatorname{arcmin}^{-2}$ (Evans et al. 1989; Pain 1996; Brainerd 1998). But, at least some, SNe may be probably related to GRBs (SN 1998bw and GRB 980425).

\subsection{AGN flares}

The spread of particular AGN flare amplitudes is large (0.1... 6.7 mag). Recently, there is growing evidence for large amplitude (more than $10 \mathrm{mag}$ ) flares on AGNs (Hudec et al. 1996). Before, the largest known QSO amplitude for 3C 279 was more than 6.7 mag (Eachus \& Liller 1975 ) but with undersampled light curve.

The expected AGNs/QSOs surface densities are $\sim(10-37) \operatorname{deg}^{-2}$ with $\lim \operatorname{mag} B / V \sim 20.5$ (Iovino et al. 1996; Hartwick \& Scade 1990) and $111 \mathrm{deg}^{-2}$ with lim mag B 22.6 (Trevese et al. 1989). Most of the QSOs are variable: the typical QSO variability in a sample of 
149 opt. selected QSOs is $0.26-0.33$ in $B 0.22-0.30$ in $R$ (Cristiani et al. 1997). There are $\sim 100$ variable QSOs $\mathrm{deg}^{-2}$ (by more than $0.1 \mathrm{mag}$ ) i.e. 1 variable QSO in $6 \times 6$ arcmin $^{2}$ brighter than $B 22.6$ (Trevese et al. 1989). $97 \%$ QSOs below B 22.5 are variable (Trevese \& Kron 1994). The QSO variability seems to increase with decreasing luminosity (Cristiani et al. 1997).

\subsection{Stellar flares}

There is growing evidence for large amplitude ( 5 mag and more) stellar flares. Flare stars detected by extended plate surveys can serve as an example: e.g. the two pairs of OTsflare stars detected by Greiner \& Motch (1995) and Hudec et al. (1997) which seem to represent large amplitude flares (5-9 mag) from otherwise typical dMe flare stars. The exact statistics of such events is however unknown.

\subsection{Variable stars}

Also variable stars may exhibit light behavior similar to OAs and OTs. The Y Dra Mira type variable star located inside the error box of the GRB 910709 detected and positioned by COMPTEL can serve as an example. The star exhibits light variations between 6 and 15 mag, with gradual light decrease after maximum (period $322 \mathrm{~d}$ ). Although the physical relation would be difficult to explain, the object shows a maximum almost exactly coincident with the GRB date. The estimated rates are available only for variable stars brighter than 20 mag: $80 \mathrm{deg}^{-2}$ for /bII/ < $20 \mathrm{deg}$ and $4 \mathrm{deg}^{-2}$ for /bII/ > $40 \mathrm{deg}$ (Hudec \& Wenzel 1996), however the discovery probability is $\sim 0.1$ (blinkmicroscope use). No statistics for variable stars below 20 mag is available (no systematic surveys). Variable stars are observed more commonly in decline than in increase since the declines are typically more slowly such as delta Cep stars, U Gem stars, flare stars, novae etc.

\subsection{OTs of unknown nature and origin}

There are real OTs of unknown nature but of astrophysical origin detected both on emulsions and CCDs. Examples (real CCD detections): (1) OT 970215. A real CCD detection with guiding error (trailing), $V 13 \mathrm{mag}$, not on any of other 800 CCD observations, nothing down 20 mag on the position, amplitude more than 7 mag (Vidal-Saiz et al. 1996). (2) OT 950806. Confirmed real object: detected on 20 CCD frames, peak magnitude I 7.5, amplitude more than 10 mag, nothing down mag 2148 hrs after detection (Toth et al. 1996).

\section{Strategy for GRB searches in optical range}

(1) it is feasible to use ground based optical devices to monitor OAs of GRBs (2) these surveys must be of wide field and high sensitivity (3) these surveys will suffer by high rate of background triggers (4) the recent SNe searches may be used to detect OAs (5) the rates of most background triggers are poorly investigated so far (6) the detected OTs must be further studied to eliminate them from background triggers.

Numerous space and ground-based experiments can be used for these purposes such as the OMC (Optical Monitoring Camera) onboard INTEGRAL $\left(5 \times 5 \mathrm{deg}^{2}\right.$, lim mag 19), CCD-based devices and telescopes (ASPA, OTM, BOOTES), digitized plate surveys and digitized archival plates.

\section{Summary}

Even the recent high localization accuracy of GRBs cannot exclude positionally coinciding OTs as just random unrelated coincidences. The exact background rate of unrelated triggers such as variable stars and extragalactic objects is unknown but may be rather high. The expected rate of faint (lim mag $B 22.6$ ) variable AGNs is 1 in a $5 \times 5$ arcmin box. The statistics of large-amplitude $(>0.5 \mathrm{mag})$ variable AGNs is unknown. The rate of faint (below mag 20) variable stars is unknown. For brighter variable stars, the expected rate is $0.03-0.5$ (depending on galactic latitude) in a $5 \times 5$ arcmin box (lim mag 20). The expected rate of faint (lim mag $R$ 23) SNe is 0.4 in a $5 \times 5$ arcmin box. The estimated total number of optically variable sources of astrophysical origin ( $\mathrm{SNe}+\mathrm{AGNs}+\mathrm{VS}$ ) is $\sim 1-2$ in a $5 \times 5$ arcmin box, lim mag 23 . It is obvious that we need a better statistics of faint variable optical objects.

Acknowledgements. The work has been supported by the Projects of the Czech Ministry for Education and Youth, Nos. ES02 and ES036.

\section{References}

Brainerd J.J., 1998, AIP Conf. Proc. 428, 545

Cristiani S., et al., 1997, A\&A 321, 123

Djorgovski S.G., et al., 1997, Nat 387, 876

Eachus L.J., Liller W., 1975, ApJ 200, L61

Evans R., et al., 1989, ApJ 345, 752

Greiner J., Motch C., 1995, A\&A 294, 177

Greiner J., et al., 1997, IAUC 6722

Hartwick F.D.A., Scade D., 1990, ARA\&A 28, 437

Hudec R., et al., 1996, in Blazar Cont. Var. ASP Conf. Ser. 110,129

Hudec R., Wenzel W., 1996, A\&ASS C707

Hudec R., et al., 1997, Proc. 2nd INTEGRAL Workshop, ESA SP-382, 481

Hudec R., et al., 1999 (this volume)

Iovino A., et al., 1996, A\&ASS 119, 265

Pain R., 1996, ApJ 473, 356

Trevese D., Kron R.G., 1994, in Multi-Wavelength Continuum Emmission of AGNs, Courvoisier T. and Blecha A. (eds.), p. 412

Toth I., et al., 1996, A\&A 315, 153

Trevese D., et al., 1989, AJ 98, 108

Vidal-Saiz J., et al., 1996, IBVS Budapest, 4324 